\title{
A WILD CANTOR SET AS THE LIMIT SET OF A CONFORMAL GROUP ACTION ON $S^{3}$
}

\author{
M. BESTVINA AND D. COOPER
}

\begin{abstract}
We construct a conformal action of a free group of finite rank on $S^{3}$ whose set of discontinuity $\Lambda$ is a wild Cantor set.
\end{abstract}

1. Introduction. This paper is a result of independent efforts by the authors to answer a question asked by M. Freedman and R. Skora in their recent manuscript [FS].

There they construct an exotic example of quasiconformal group action on $S^{3}$ whose limit set is a wild Cantor set. Some interesting features of that example are that each group element is topologically conjugate to a loxodromic conformal diffeomorphism of $S^{3}$, but the entire group is not topologically conjugate to a conformal group.

Below we construct a conformal group $G$ (abstractly a free group of finite rank) whose limit set is a wild Cantor set. As opposed to the Freedman-Skora example, the group $G$ will contain lots of parabolic conformal diffeomorphisms.

Consequently, it has been conjectured that a wild Cantor set cannot be the limit set of a conformal free group action with no parabolics.

For the sake of brevity, we abuse notation slightly by writing $\bigcup C$ for $\bigcup_{C \in C} C$, where $C$ is a finite collection of subsets of $\mathbf{R}^{3}$ or $S^{3}$. We define

$$
\operatorname{mesh} C=\max \{\operatorname{diam} C \mid C \in C\}
$$

where metrics on $\mathbf{R}^{3}$ and $S^{3}$ are standard.

2. The example. Our example is modeled on the standard Schottky action (cf. [Ch, Ma]), except that we allow defining spheres to touch.

By a pair of eyeglasses we mean the compactum $E$ consisting of two disjoint simple closed curves $S_{1}, S_{2}$ joined by an arc $A$. Consider the embedding of $E$ in $\mathbf{R}^{3}$ defined by Figure 1 (the Hopf link plus an arc joining the components).

Now let $C$ be a collection of small round balls placed along $E \subset \mathbf{R}^{3}$ so that adjacent balls touch (see Figure 2). Note that most elements of $C$ will have two points of contact. There are two exceptional elements $T_{1}, T_{2}$ that contain nonmanifold points of $E$; they have three points of contact. Note that balls along the circular parts $S_{1}, S_{2}$ of $E$ do not separate between their contact points, while those along $A$ separate between their contact points.

Let $\varphi: C \rightarrow C$ be a fixed point-free involution $(\varphi \cdot \varphi=I d)$ such that

(i) $\varphi\left(T_{1}\right)=T_{2}$; and

Received by the editors February 24, 1986.

1980 Mathematics Subject Classification (1985 Revision). Primary 20H10, 57M30, 57S99.

Key words and phrases. Conformal group action, limit set, wild Cantor set.

The first author was supported in part by NSF Grant DMS 8513582. 


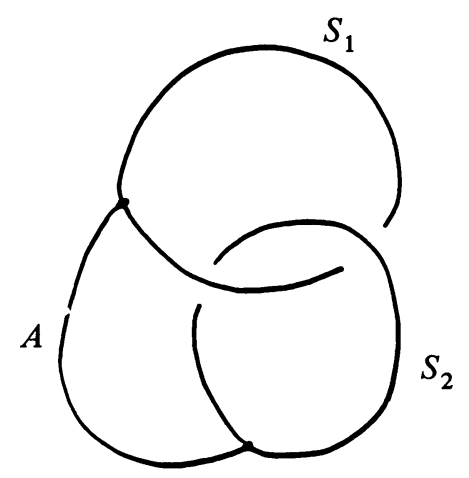

FIGURE 1

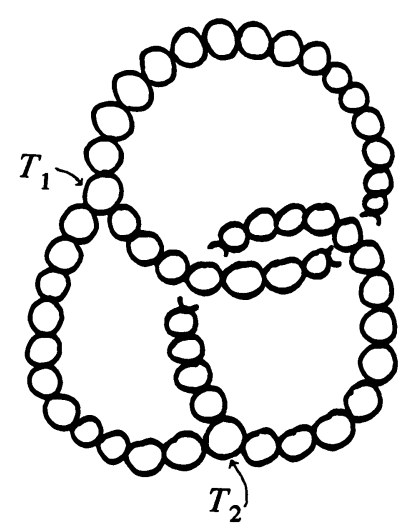

FIGURE 2

(ii) along each circular part $S_{1}, S_{2}$ of $E$ there are at least two balls $C^{\prime}, C^{\prime \prime}$ such that $\varphi\left(C^{\prime}\right), \varphi\left(C^{\prime \prime}\right)$ lie along $A$.

The collection $C$ can be transferred via the inverse of the stereographic projection to $S^{3}$. Suppressing the stereographic projection, we have $E \subset S^{3}, C=$ collection of round balls in $S^{3}$. For each $C \in C$ choose a conformal diffeomorphism $h_{C}: S^{3} \rightarrow S^{3}$ so that

(iii) $h_{C}(C)=S^{3}-\operatorname{int} \varphi(C)$;

(iv) $h_{C}$ maps the points of contact of $C$ to the points of contact of $\varphi(C)$; and

(v) $h_{\varphi(C)}=h_{C}^{-1}$.

Let $G$ be the group of conformal diffeomorphisms of $S^{3}$ generated by $\left\{h_{C} \mid C \in C\right\}$. If the collection $C$ consisted of disjoint balls, $G$ would be the classical Schottky action, whose limit set is a tame Cantor set. The same arguments apply to our case to show that

(1) $G$ is (abstractly) a free group of finite rank,

(2) $G$ acts freely and properly discontinously in the complement of its limit set $\Lambda$,

(3) $\Lambda=\bigcap_{n=0}^{\infty}\left(\bigcup C_{n}\right)$, where $C_{0}=C$ and $C_{n+1}=\left\{h_{C}\left(C_{n}\right) \mid C \in C, C_{n} \in\right.$ $C_{n}, h_{C}\left(C_{n}\right)$ is contained in an element of $\left.C_{n}\right\}$. Also, mesh $C_{n} \rightarrow 0$ as $n \rightarrow \infty$. 


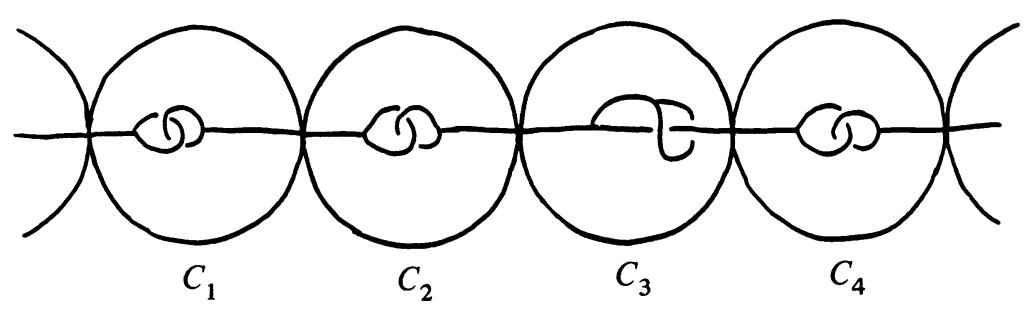

FIGURE 3

In Figure 3 we have drawn a part of the "core" of $U C_{1}$. In the picture, we assumed that $\varphi\left(C_{1}\right), \varphi\left(C_{2}\right), \varphi\left(C_{4}\right)$ lie along the arc $A$, while $\varphi\left(C_{3}\right)$ lies along a circular part of $E$.

The main result of this paper is

\section{THEOREM. $\Lambda$ is a wild Cantor set.}

ProOF. We first show that $\Lambda$ is totally disconnected, i.e. that it does not contain any nondegenerate continuum $K$. Assuming $\operatorname{diam} K>\varepsilon>0$, choose $n \in \mathbf{N}$ so that mesh $C_{n}<\varepsilon / 2$. Since $K \subseteq \Lambda \subseteq \cup C_{n}$, it follows that there is $C_{n} \in C_{n}$ such that $K \cap C_{n}$ spans between two different contact points of $C_{n}$. Find (unique) $g \in G$ (which will be a word in $h_{C}$ 's of length $n$ ) and $C_{0} \in C_{0}=C$ such that $g\left(C_{0}\right)=C_{n}$. Then $K^{\prime}=g^{-1}\left(K \cap C_{n}\right)$ is a compactum in $C_{0} \cap \Lambda$ that spans between two different contact points of $C_{0}$.

Since $K^{\prime} \subseteq C_{0} \cap\left(\bigcup C_{1}\right)$, it follows that $h_{C_{0}}\left(K^{\prime}\right) \subseteq\left(S^{3}-\operatorname{int} \varphi\left(C_{0}\right)\right) \cap\left(\bigcup C_{0}\right)$. If $\varphi\left(C_{0}\right)$ lies along $A$, then the latter set does not span between contact points of $\varphi\left(C_{0}\right)$, while $h_{C_{0}}\left(K^{\prime}\right)$ does, a contradiction. If $\varphi\left(C_{0}\right)$ lies along a circular part $S_{i}$ of $E$, then $h_{C_{0}}\left(K^{\prime}\right) \cap C$ spans between contact points of $C$ for every $C \in C$ along $S_{i}$ (this statement also holds for $C=T_{i}$ with obvious interpretation).

In particular, by property (ii), we find $C_{0}^{\prime} \in \mathcal{C}$ such that $\varphi\left(C_{0}^{\prime}\right)$ lies along $A$, and $h_{C_{0}}\left(K^{\prime}\right) \cap C_{0}^{\prime}$ spans between contact points of $C_{0}^{\prime}$. Then we arrive at a contradiction just like in the previous paragraph.

Since each $C_{n} \in C_{n}$ contains $\operatorname{card}(C)-1$ balls of $C_{n+1}$, it is clear that $\Lambda$ has no isolated points. Therefore, $\Lambda$ is a Cantor set.

It remains to establish the wildness of $\Lambda$. We show that $S^{3}-\Lambda$ is not simplyconnected.

$D=S^{3}-\left(\bigcup_{C \in C}\right.$ int $C \cup$ points of contact $)$ is a fundamental domain for the action of $G$ on $S^{3}-\Lambda$. Notice that the action of $G$ can naturally be extended to an action on $\mathbf{H}^{4} \cup S^{3}$, the compactified 4-dimensional hyperbolic space, via isometries of $\mathbf{H}^{4}$. The extended action has the same limit set $\Lambda$.

Consider the commutative diagram

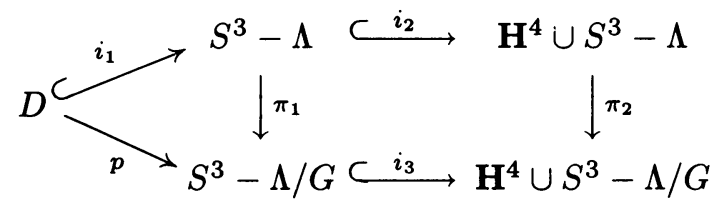

where the vertical maps are natural projections. 
Observe that $D$ has the homotopy type of the wedge of two circles, and therefore $\pi_{2} i_{2} i_{1}=i_{3} p$ is not injective on the fundamental groups. On the other hand, if $S^{3}-\Lambda$ is simply-connected, $i_{3}$ induces an isomorphism on the fundamental groups. To get a contradiction, we show that $p$ is injective on the fundamental groups.

$S^{3}-\Lambda / G$ is a 3-manifold that can be obtained from $D$ by gluing $\partial_{-} C=\partial C \cap D$ with $\partial_{-} \varphi(C)=\partial \varphi(C) \cap D$ via $h_{C}$ for $C \in C$, and $p$ is the natural quotient map. Notice that, $\partial_{-} C \subseteq D$ is injective on the fundamental groups for every $C \in \mathcal{C}$ (a small linking circle around $A \subset E \subset S^{3}$ represents the commutator $[x, y]$ of the free generators $x, y$ of $\left.\pi_{1}(D)\right)$, and therefore each identification $\partial_{-} C \equiv \partial_{-} \varphi(C)$ corresponds to an HNN extension of the fundamental group. Since a group injects into an HNN extension of itself, the result follows.

\section{REFERENCES}

[Ch] V. Chuckrow, Schottky groups and limits of Kleinian groups, Bull. Amer. Math. Soc. 73 (1967), 139-141.

[FS] M. Freedman and R. Skora, Strange actions of groups on spheres (preprint).

[Ma] B. Maskit, A characterization of Schottky groups, J. Analyse Math 19 (1967), 227-230.

Department of Mathematics, University of California at LOS ANGeles, Los ANGELES, CALIFORNIA 90024 (Current address of M. Bestvina)

Department of Mathematics, University of California at San Diego, LA JOLla, CALIFORNia 92093

Current address (D. Cooper): Department of Mathematics, University of Minnesota, Minneapolis, Minnesota 55455 